\title{
The 5xFAD mouse model of Alzheimer's disease
}

\section{Caroline Ismeurt, Patrizia Giannoni and Sylvie Claeysen [1]}

\section{[1] Corresponding author:}

\section{Dr Sylvie Claeysen}

IGF

University of Montpellier, CNRS, INSERM

141 rue de la Cardonille

Montpellier

France

Telephone: +33 (0)434359215

Email: sylvie.claeysen@igf.cnrs.fr

Additional email: sylvie.claeysen@gmail.com

\section{Dr Patrizia Giannoni}

University of Nîmes

Rue du Dr Georges Salan

Nîmes

France

Telephone: +33 (0)4 66279571

Email: patrizia.giannoni@unimes.fr

Additional email: patrizia.giannoni@gmail.com

\section{Caroline Ismeurt}

IGF

University of Montpellier, CNRS, INSERM

141 rue de la Cardonille

Montpellier

France

Telephone: +33 (0)434359215

Email: caroline.ismeurt@igf.cnrs.fr

Additional email: caroline.ismeurt@outlook.com

Departmental Contact (for communications in absentia)

Ms Aurore Castillo

IGF

University of Montpellier, CNRS, INSERM

141 rue de la Cardonille

Montpellier

France

Telephone: +33 (0)4 34359243

Email: aurore.castillo@igf.cnrs.fr

\section{Word count}

Total words: 5998 (without abstract and key words)

6 figures

52 references

2044 words in references

\section{Short title}

$5 x F A D$ mice 


\begin{abstract}
During the past 20 years, a plethora of transgenic mouse models have been developed in order to reproduce the main features of Alzheimer's Disease (AD). Due to the complexity of the disease, no ideal animal model exists to date. The 5xFAD mouse line recapitulates numerous neuropathological insults and cognitive alterations found in human AD patients: from amyloid deposits, intense gliosis and neuroinflammation, including neurotransmission alteration, synaptic loss and neurodegeneration to behavioural and memory deficits. The extremely aggressive amyloid pathology of these mice makes them a suitable model for early-onset AD. This chapter describes and illustrates the main characteristics of the 5xFAD mouse model and discusses its usefulness in the context of pre-clinical evaluation of drug-candidates or promising therapeutic targets against AD.
\end{abstract}

\title{
Key words
}

Transgenic mouse; amyloid pathology; neurovascular damage; neuroinflammation; behavioural deficits 


\author{
List of abbreviations \\ $\boldsymbol{A} \boldsymbol{\beta}$. Amyloid $\beta$ peptide \\ AD. Alzheimer's disease \\ $\boldsymbol{A P P}$. Amyloid precursor protein \\ BACE1. $\beta$-secretase \\ $\boldsymbol{B D N F}$. Brain-derived neurotrophic factor \\ $\boldsymbol{C A A}$. Cerebral amyloid angiopathy \\ $\boldsymbol{F A D}$. Familial Alzheimer Disease \\ $\boldsymbol{G F A P}$. Glial fibrillary acidic protein \\ Iba1. Ionized calcium-binding adapter molecule 1 \\ IL. Interleukin \\ LTP. Long term potentiation \\ NFTs. Neurofibrillary tangles \\ $\boldsymbol{P H F}$. Paired helical filaments \\ PSEN1. Presenilin 1 \\ $\boldsymbol{T G F} \boldsymbol{\beta}$. Transforming growth factor $\beta$ \\ $\boldsymbol{T L R}$. Toll-like receptor \\ $\mathbf{T N F} \boldsymbol{\alpha}$. Tumor necrosis factor $\alpha$
}

\title{
Introduction
}

Many transgenic mouse models have been developed to reproduce the numerous brain features of Alzheimer's disease (AD), ranging from amyloid- $\beta(\mathrm{A} \beta)$ peptide deposition, neurofibrillary tangles, neuro-inflammation and synaptic alterations to neuronal loss (Selkoe and Schenk, 2003). As wild-type mice develop neither plaques nor tangles in their lifespan, the introduction of human genes bearing mutations involved in familial forms of the disease was a key step to recapitulate AD pathology (Oakley et al., 2006). The heterologous mouse line Tg6799, also named 5xFAD, expresses five Familial Alzheimer's Disease mutations under the control of the neuron-specific thyl promoter: three mutations on human amyloid protein precursor (APP695; London, Swedish and Florida) and two mutations on human presenilin-1 (PS1; M146L and L286V) (Figure 1). No human tau transgene is expressed in this mouse line. The 5xFAD mouse line is the transgenic mouse model of AD presenting the earliest onset and most intense amyloid pathology. Interestingly, this mouse model recapitulates quite all the neuropathological features associated with AD: amyloid plaques, gliosis, cognitive impairment, neurotransmission defects, synaptic loss and neurodegeneration (Figure 2). The only missing features are the tau tangles, even if some reports are contradictory on this point. The AD-like pathology in 5xFAD mice can be divided in three phases as in human AD. The asymptomatic phase, around 2 months of age, is characterized by the presence of amyloid plaques and appearance of gliosis (Figure 2). The prodromal phase with extensive amyloid deposits and gliosis is defined by the appearance of cognitive deficits around 4 months of age. The symptomatic phase starts around 6 months of age and over with extreme amyloid pathology and associated neuroinflammation, marked cognitive deficits and neurotransmission impairments. Finally, late AD-like stages or "severe AD-like pathology" are characterized by synaptic and neuronal loss around 9 months of age. This chapter describes all the neuropathological and behavioural features of the 5xFAD mouse model to highlight its interest for fundamental research and pre-clinical studies. 


\section{Amyloid pathology}

The overproduction and aggregation of $A \beta$ in the brain are major pathological hallmarks of $A D$. $\mathrm{A} \beta$ is produced and released in the extracellular domain through the amyloidogenic pathway of APP processing. This pathway involves two successive cleavages by proteases: BACE1 and $\gamma-$ secretase. The cleavage by $\gamma$-secretase is not precise and leads to several A $\beta$ peptides with different lengths including $A \beta_{1-40}$ (40 amino acids) and $A \beta_{1-42}$ (42 amino acids), the two main $\mathrm{A} \beta$ isoforms. In the $5 \mathrm{xFAD}$ mouse model, the three FAD mutations on the human APP transgene are located around the cleavage site of $\beta$ - and $\gamma$ - proteases and are involved in the overproduction of either total $A \beta$ or specifically $A \beta_{1-42}$ peptides. The London (V717I) and Florida (I716V) mutations, located around the $\gamma$-secretase cleavage site, have been reported to induce an increase of the $A \beta_{1-42} / A \beta_{1-40}$ ratio (De Jonghe et al., 2001, Herl et al., 2009). The Swedish mutation (K670N/M671L), close to the BACE1 cleavage site, has been reported to induce an increase of total A $\beta$ (Hsiao et al., 1996). The two FAD mutations present on the PSEN1 transgene (catalytic subunit of $\gamma$-secretase) have been shown to also increase the $A \beta_{1-42} / A \beta_{1-40}$ ratio (Scheuner et al., 1996, Citron et al., 1997). Due to a constitutive expression of the transgene, an overproduction of $\mathrm{A} \beta$ is detectable as early as 1.5 months of age in $5 \times \mathrm{FAD}$ mouse brains. This amyloid accumulation corresponds mainly to soluble $A \beta$ peptides $\left(A \beta_{1-40}\right.$ and $\left.A \beta_{1-42}\right)$. At the age of 2 months, $A \beta$ oligomers aggregate in $A \beta$ fibrils and diffuse amyloid plaques, while forming dense amyloid plaques later on. The amyloid pathology begins in the deep cortical layers of the brain and subiculum. Then, with aging of the mice, plaques spread to cover much of the cortex and hippocampus (Schaeffer et al., 2011) (Figure 3). This increase in plaque number and density reaches a plateau at 10 months of age in male $5 x F A D$ mice, whereas in females the maximal density is not even reached at 14 months of age (Bhattacharya et al., 2014). This amyloid load has deleterious effects on the brain including the development of a persistent neuroinflammation, neuronal loss, cognitive deficits and inhibition of LTP.

\section{Neuroinflammation}

Resident microglia and astrocytes, which are innate immune cells of the brain, are clustered around amyloid plaques (Perlmutter et al., 1990) and are mediators of the neuroinflammation commonly observed in AD (Figure 4). In a healthy brain, microglia is in a resting state and surveys the surrounding area. In the $5 \mathrm{xFAD}$ mouse brain, the $\mathrm{A} \beta$ pathology induces the activation of microglia into an anti-inflammatory state, called M2. This activation is mediated mainly by toll-like receptor 2 (TLR2) that recognizes $A \beta_{1-42}$ (Liu et al., 2012). M2 microglia releases anti-inflammatory cytokines such as IL-10 and TGF $\beta$ (Orihuela et al., 2016) and has a phagocytic activity that contributes to the clearance of $A \beta$ oligomers. Nevertheless, as the amyloid pathology is persistent, microglial cells fail to clear all $\mathrm{A} \beta$ peptides and transit chronically into a pro-inflammatory state, called M1. Several cytokines, such as IL-6, IL- $\beta$ and $\mathrm{TNF} \alpha$, are released inducing a persistent neuroinflammation, which aggravates brain damages (Spangenberg et al., 2016). This pro-inflammatory environment is believed to appear around the age of 2 months when the amyloid deposits have arisen. However, the microglia activation may occur before (Boza-Serrano et al., 2018). The astrocytes, the second type of immune cells in the brain, are activated in a proportional manner to the level of $A \beta_{1-42}$ and amyloid plaques (Oakley et al., 2006). This activation is mediated by C1q, one component of the complement system, and induces the transition of astrocytes into reactive astrocytes (Fonseca et al., 2004). The latter have a phagocytic activity to clear $\mathrm{A} \beta$ peptides and produce neurotrophic factors such as BDNF (Kimura et al., 2006, Wyss-Coray et al., 2003). However, reactive astrocytes also produce proinflammatory cytokines (Johnstone et al., 1999) and with aging they present impairments in clearing $A \beta$ peptides and in neuronal support (Iram et al., 2016). This alteration of astrocyte function participates to the maintenance of the chronic neuroinflammation and brain damages. 


\section{Behavioural aspects of the model}

The 5xFAD mouse model develops different types of memory deficits around the age of 4 months when amyloid plaques and neuroinflammation are present (Oakley et al., 2006). In many tests involving hippocampus-dependent memory function such as Morris water maze, Y-maze, fear conditioning and object or social recognition, 5xFAD mice have demonstrated impaired performances (Hongpaisan et al., 2011, Jawhar et al., 2011). Working memory (short or long term) is altered in 5xFAD mice as early as 4 months of age (in the B6/SJL background) (Giannoni et al., 2013) (Figure 5). An impairment of frontal cortex function has also been demonstrated at 4 months of age using the olfactory H-maze and the delayed reaction paradigm (Girard et al., 2013). Associative learning memory is also affected in this mouse model but only around the age of 6 months (Baranger et al., 2017, Roddick et al., 2014). Cognitive performances of 5xFAD mice in conditioned taste aversion and contextual fear conditioning paradigms are impaired around 6 months of age and 9 months of age respectively (Devi and Ohno, 2010). As noticed in the Jackson Laboratory depository (https://www.jax.org/strain/006554) and in comparison to the original B6/SJL-5xFAD strain, the phenotype in the $5 \mathrm{xFAD}$ mice is less pronounced when the mice are backcrossed on $\mathrm{C} 57 \mathrm{BL} / 6 \mathrm{~J}$ background, the more commonly used laboratory mouse strain to perform cognitive assessment. Indeed, we have experienced around one-month delay in the phenotype when using C57BL/6J-5xFAD mice compared to the original B6/SJL-5xFAD mice (Oakley et al., 2006). Amyloid pathology is delayed and thus the first cognitive deficits appear around 4.5 to 5 months of age. The status of the animal housing facility is another parameter to consider. It has recently been demonstrated that gut microbiota composition impacts the amyloid pathology development and that AD-mice breaded in conventional microbiota have more amyloid in the brain than AD-mice born in germ-free environment (Brandscheid et al., 2017, Harach et al., 2017). Consequently, if the mice are breaded in Specific Pathogen Free (SPF) or Specific and Opportunist Pathogen Free (SOPF) facilities, the behavioural phenotypes could be delayed due to a less aggressive amyloid pathology.

\section{Synaptic plasticity and LTP}

According to the amyloid cascade hypothesis, amyloid accumulation in the AD brain induces numerous neuropathological changes that precede neurodegeneration (Hardy \& Selkoe, 2002). Long-term potentiation (LTP) is a perfect read-out of cellular communication processes that underlie learning and memory. In 5xFAD mice, phenotypic differences between plaque deposits in the cortex and hippocampus (more dense plaque in the latter) were linked to differences in LTP in these areas (Crouzin et al., 2013). Within the hippocampus of 5xFAD mice, an intense astrogliosis in the dentate gyrus, compared to CA1 region, was associated with enhanced tonic GABA (gamma aminobutyric acid) current mediated by $\alpha 5-\mathrm{GABA}_{\mathrm{A}}$ receptors in dentate granule cells (Wu et al., 2014). Blockade of these $\mathrm{GABA}_{\mathrm{A}}$ receptors rescued LTP deficits and restored working memory performances in the Y-Maze test. Restoration of LTP in 5xFAD mice is thus an important parameter to consider in the evaluation tests of candidate molecules or actors as promising therapeutic targets against AD (Baranger et al., 2016, MacPherson et al., 2017).

\section{Vascular pathology}

In recent years, the impact of the cerebral vasculature on the pathophysiology of AD has gained attention. Indeed, a link has been evidenced between AD development and vascular risk factors such as hypertension, obesity, type 2 diabetes, stroke and atherosclerosis (Cechetto et al., 2008). An efficient brain vasculature is necessary for a correct delivery of nutrients and oxygen, but it's also fundamental for its role of detoxification and barrier. Brain vessels help in clearing possible toxic elements, such as the amyloid peptides responsible of plaque formation, and modulate the substances that access the brain thanks to the blood brain barrier (BBB). This finely organized structure is composed of tight junctions between endothelial cells that strictly control the 
movements of molecules between the blood and the brain. Several elements, including aging, genetic and epigenetic factors, but also a number of pathologies, determine an impairment of brain vasculature efficiency, with consequent accumulation of toxic species and alteration of brain homeostasis. The $5 \mathrm{xFAD}$ mouse model has been demonstrated to accurately reproduce the alterations of brain vasculature typical of AD presymptomatic and symptomatic stages (Giannoni et al., 2016). Thanks to a longitudinal study conducted with in-vivo two-photon microscopy associated to classical post-mortem analysis, it was evidenced that $5 \mathrm{xFAD}$ mice accumulate amyloid around vessels in what is defined as cerebral amyloid angiopathy (CAA), starting at 3 months of age and accumulating overtime (Figure 6A). The study confirms a precedent observation of BBB disruption (Kook et al., 2012) near amyloid deposits (Figure 6A, B, C), also associated with matrix-metalloproteases secretion. Indeed, although the association between CAA and cognitive performances is still discussed, the majority of human AD cases show this feature. Another set of cells participating to the neurovascular unit and controlling vessels efficiency and contraction, the pericytes, are also impacted in 5xFAD mice. Their expression level changes overtime becoming more important at 9 and 12 months of age, considered late stages AD in this model. To note, their increased level of expression seems associated with their redistribution, although further studies are needed to better characterize this phenomenon. Pericytes have been proposed as major players in the reparation process after a brain insult and are currently under investigation both in human $\mathrm{AD}$ cases and mouse models as they represent a well-promising target of intervention. Finally, 5xFAD mice reproduce one of the main aspects contributing to $\mathrm{AD}$ development: vascular inflammation. A strong microglial activation associated with astrogliosis is evidenced starting at 3 months of age around brain vessels and in strict association with amyloid accumulation and vessels damage. Clusters of reactive inflammatory cells were found correlating with disruption of tight junctions detected by leakages of FITC-albumin. Changes in the expression of inflammation markers (e.g. IL-6) have also been observed in this model (Gurel et al., 2018), probably contributing to vascular inflammation. As for the human pathology, vascular inflammation aggravates with aging in the $5 \mathrm{xFAD}$ mouse model.

\section{Neuronal loss/synaptic dysfunctions}

The 5xFAD mouse model reproduces the neuronal loss and synaptic dysfunctions that characterize human AD. This feature is particularly interesting, as many other AD animal models do not present any neuronal damages. Interestingly, neuronal loss is particularly evident in the hippocampus and in cortex areas involved in memory formation and processing (Eimer and Vassar, 2013). A profound loss of layer 5 pyramidal neurons is observed in 5xFAD mice starting at 12 months of age (Oakley et al., 2006), while other alterations such as axonal dystrophy, loss of spines and basal dendrites (Buskila et al., 2013) appear much earlier, around 4-6 months. As behavioural deficits appear around 4 months in this model, such early changes are thought to impact cognitive performances of the mice before late stage neuronal death occurs. Thanks to electrophysiological studies of such neurons, a direct link between intraneuronal A $\beta$ accumulation, that precedes plaque formation, and neuronal loss has been hypothesized. Presynaptic vesicular machinery, as well as postsynaptic effects, have been underlined, probably due to the impact of $A \beta$ peptides on glutamatergic conductance. A general consensus is present in the actual literature that considers the presence of amyloid as responsible for synaptic dysfunctions (Marchetti and Marie, 2011) and neuronal loss (Eimer and Vassar, 2013).

\section{Tau pathology}

Although the $5 x F A D$ model is considered one of the most exhaustive concerning the reproduction of $\mathrm{AD}$ signs, the presence of neurofibrillary tangles (NFTs) is still discussed. While the first description of the 5xFAD model reported the lack of NFTs (Oakley et al., 2006), other more recent papers show a positive staining of PHF (paired helical filaments) tau sites Ser202 
and Thr205 with the AT8 antibody, and identify NFTs in the cortex and hippocampus of female 5xFAD mice of 6-8 months of age (Tohda et al., 2012). The antibody used being directed against the human tau, this supposes a cross-reaction with the mouse form. Hyperphosphorylation of tau seems confirmed in 5xFAD mice (Grinan-Ferre et al., 2016), as stated by mRNA analysis. However, the work of Maarouf and colleagues evidenced no neurofibrillary tangles with AT8 antibody (Maarouf et al., 2013). Possible causes of heterogeneity in the observed results could be the age, sex and genetic background of animals, as well as methodological and analysis differences (immunohistochemistry protocol, microscope...). Discrepancies among results point to the need of further investigations.

\section{Gender issue}

In the human population, the prevalence of $\mathrm{AD}$ is higher among women than among men with a 2:1 women/men ratio. A detailed review of current knowledge on sex-related differences in human AD has been recently conducted (Ferretti et al., 2018). This excellent work pointed out sex differences in AD patients not only at an epidemiological point of view, but also in clinical manifestations, biomarkers patterns, risk factors and treatment performance. Clearly, researchers have now to consider gender issues in the deciphering of molecular and mechanistic comprehension of $\mathrm{AD}$ in preclinical research. A higher $\mathrm{A} \beta$ production and deposition has been reported in female 5xFAD mice compared to male mice of the same age (Bhattacharya et al., 2014, Oakley et al., 2006). This feature was also reported in other transgenic AD mouse models such as APP/PS1, or 3xTg-AD (Callahan et al., 2001, Wang et al., 2003). Of interest, despite an increased $\mathrm{A} \beta$ burden, female 5xFAD mice (SJL background strain) have a similar lifespan than males, whereas in 3xTg-AD mice an early mortality in males has been reported (Rae and Brown, 2015). External environment can also induce gender-related pathology as stress increases $A \beta$ levels in female 5xFAD but not in males (Devi et al., 2010). Female 5xFAD mice present better olfactory performance than males in olfactory working memory task (Roddick et al., 2014). However, odour detection performance may vary in $5 \mathrm{xFAD}$ depending on the concentration of the odorant molecules (Roddick et al., 2016). As in humans, treatment efficiency can differ between males and females 5xFAD. Vitamin D supplementation is efficient only when administered during the symptomatic phase for the females (Landel et al., 2016) and only in the early asymptomatic phase for the males (Morello et al., 2018). We experienced also differences in treatment efficiency when a serotonin type 4 receptor agonist was chronically administrated to $5 \mathrm{xFAD}$ mice. Two-month treatments are sufficient to prevent cognitive deficits in females, whereas longer treatments were necessary to reach similar effects in males (Baranger et al., 2017, Giannoni et al., 2013). Collectively, there is a crucial need to systematically investigate sex differences in mouse models of $\mathrm{AD}$ with adapted sizes of groups in order to obtain an accurate statistical power.

\section{Drawbacks of the 5xFAD mouse model}

In this transgenic mouse, the extremely aggressive $A \beta$ pathology, with extensive extracellular plaque formation beginning at 2 months of age, is definitely not in accordance with the time course of human sporadic AD. The slow progressive pathophysiological development of clinical $\mathrm{AD}$ in patients is not accurately reproduced in this transgenic mouse model. New models, using injections of adeno-associated viruses in rodents, have been developed to mimic more precisely the initial stages and the progression of $\mathrm{AD}$ in patients, by controlling the expression in time and space of the transgenes (Audrain et al., 2016).

\section{Advantages of the 5xFAD mouse model}

The extreme rapidity of the amyloid neuropathology and associated behavioural deficits make this transgenic mouse a proper research model for early onset AD (Jawhar et al., 2012, Oakley 
et al., 2006). Moreover, the appearance of cognitive deficits as early as 4 months of age renders the $5 \mathrm{xFAD}$ mice suitable for evaluating the preventive potential of chronic treatments with disease-modifying molecules (Baranger et al., 2017, Giannoni et al., 2013). Indeed, if the chronic administration of a compound is able to slow $A \beta$ production and delay the appearance of cognitive impairments in $5 \mathrm{xFAD}$ mice, that produce the highest level of amyloid, the same compound should be efficient in transgenic mouse models presenting moderate $A \beta$ accumulation. Additionally, such a rapidity to obtain relevant and measurable features in the model induces a reduction in time (and costs) involving animal housing and protocol-related expenses. The ethical aspect of such improvement is a particularly important argument promoting the use of this mouse model.

\section{Conclusion}

To conclude, 5xFAD mice represent the more rapid and intense transgenic mouse model of $\mathrm{AD}$, in terms of amyloid pathology. This model recapitulates numerous features of clinical AD in human patients such as amyloid deposits, gliosis, synaptic degeneration, working memory impairment, reduced anxiety, and selective neuronal loss. Besides controversies due to the early and aggressive plaque development phenotype, this model remains of great interest to preclinically evaluate the disease-modifying potential of new compounds against AD.

\section{Definitions}

Amyloid plaques. Extracellular aggregates mainly composed of neurotoxic amyloid peptides and found in post-mortem brains of Alzheimer's disease patients. With aging, plaques can also appear in the brains of cognitively healthy people.

Neurofibrillary tangles. Intracellular fibrillary structures composed of hyperphosphorylated tau protein. Theses aggregates are a marker of Alzheimer's disease but are also found in tauopathies.

Complement system. Belong to the immune system and correspond to plasma proteins that recognize pathogens and participate to their elimination.

Novel object recognition test. Memory test including a training phase where the mouse can explore two similar objects and a restitution phase in which one of the objects is replaced by a novel object. The amount of time between the training and restitution phase allows to analyse the short-term memory (i.e. 1 hour) or the long-term memory (i.e. 24 hours).

Pericytes. Perivascular cells involved in the regulation of capillary blood flow and maintenance of the blood-brain barrier.

\section{Key facts of neurovascular pathology}

- A healthy vascular system is mandatory for proper functioning of the brain.

- Amyloid accumulation around vessels (cerebral amyloid angiopathy or CAA) induces vessel damage in Alzheimer's disease.

- Vessel breaks in Alzheimer's disease are evidenced by leaks of immunoglobulins in the brain.

- An intense vascular inflammation accompanies vessel damage.

- The neurovascular unit is remodelled in Alzheimer's disease with redistribution of the pericytes. 


\section{Summary points}

- The 5xFAD mouse model reproduces the majority of Alzheimer's disease hallmarks, including amyloid plaques, neuronal death, synaptic dysfunctions, cerebral amyloid angiopathy and vessels alterations associated to inflammation.

- As for human Alzheimer's disease, the gender issue should be considered in the 5xFAD mouse model, being the pathology more aggressive in females than in males.

- The rapid and aggressive progression of the pathology in 5xFAD mice is not in accordance with the slow development of sporadic Alzheimer's disease in humans.

- The 5xFAD mouse line is a proper research model for early onset Alzheimer's disease.

- This animal model enables a time reduction of the experimental protocols and a diminution of the research costs.

\section{Acknowledgements}

The authors would like to thank Dr Nicola Marchi for the gift of vasculature images. Supported by: CNRS, INSERM, Montpellier University, French Research National Agency ANR (ANR12-BSV4-008-01 ADAMGUARD), Association France Alzheimer, Fondation Vaincre Alzheimer, Région Languedoc Roussillon and Fondation Plan Alzheimer. Confocal MRI platform (Montpellier, France).

\section{Titles and legend of figures}

Title of Figure 1: Schematic representation of 5xFAD transgenes.

Legend to Figure 1: Arrows indicate the familial Alzheimer's disease mutations in human amyloid precursor protein (APP) and presenilin-1 (PS1) transgenes that are under the control of the neuron-specific thy 1 promotor. Coding regions are depicted. For a more complete view of the untranslated regions see (Oakley et al., 2006).

Unpublished scheme.

Title of Figure 2: Schematic representation of pathology time course in the 5xFAD mouse model.

Legend to Figure 2: Key Alzheimer's disease features that are reproduced by the mouse line are summarized in accordance with the time scale. The putative correspondence with clinical disease is indicated. As controversial in the model, tau pathology is not indicated on this picture.

Unpublished scheme.

Title of Figure 3: Amyloid plaques in the 5xFAD mouse model.

Legend to Figure 3: Representative images of $30 \mu \mathrm{M}$ sagittal brain sections where plaques are stained with Thioflavin-S in the frontal cortex (A) and entorhinal cortex (B). I-VI : cortical layers I to VI; FCx: Frontal Cortex; Ect: Ectorhinal area; PRh: Perirhinal area; LEnt: Entorhinal area, lateral part.

Unpublished data.

Title of Figure 4: Microgliosis and astrogliosis in the 5xFAD mouse model.

Legend to Figure 4: Representative images of $30 \mu \mathrm{M}$ sagittal brain sections in the CA1 region of the hippocampus. (A) Ibal staining of microglia. (B) GFAP staining of astrocytes. 
Title of Figure 5. Learning performance of 3 and 5-month-old WT and 5xFAD mice assessed with the Novel Object Recognition test.

Legend to Figure 5: The 5XFAD mice are deficient at 5 months of age as indicated by the lower percentage of time devoted to the exploration of the novel object in comparison to the WT mice. Retention interval: 24 hours. 5-8 mice per group. Data are the mean \pm SEM. $* * * p<0.001$. Unpublished data.

Title of Figure 6: Vascular pathology of the 5xFAD mouse model at 12 months of age.

Legend to Figure 6: A: Amyloid accumulation (red) is stained using 6E10 anti-A $\beta$ antibody; vasculature (green) is evidenced using fluorescent-albumin staining (FITC-albumin). With aging, 5xFAD mice present vascular amyloid deposits (arrows) and intense parenchymal A $\beta$ deposits, including dense plaques (arrow heads) and diffuse plaques (puncta). Vascular deposits of $A \beta$ induce vessel damages (star) as evidenced by leakages of FITC-albumin staining. B: Larger view of cortical vasculature (white) and leakages of FITC-albumin. No leakages are detectable in WT aged mice $(\mathrm{C}$, upper left panel) compared to 5xFAD of the same age $(\mathrm{C}$, upper right panel). $\mathrm{C}$, lower panel: higher magnification of vessel damage and leakage in 5xFAD mice. Unpublished data. Courtesy of Dr Nicola Marchi (IGF, Montpellier).

\section{References}

Audrain, M., Fol, R., Dutar, P., Potier, B., Billard, J. M., Flament, J., Alves, S., Burlot, M. A., Dufayet-Chaffaud, G., Bemelmans, A. P., Valette, J., Hantraye, P., Deglon, N., Cartier, N. \& Braudeau, J. 2016. Alzheimer's disease-like APP processing in wild-type mice identifies synaptic defects as initial steps of disease progression. Mol Neurodegener, 11, 5.

Baranger, K., Giannoni, P., Girard, S. D., Girot, S., Gaven, F., Stephan, D., Migliorati, M., Khrestchatisky, M., Bockaert, J., Marchetti-Gauthier, E., Rivera, S., Claeysen, S. \& Roman, F. S. 2017. Chronic treatments with a 5-HT4 receptor agonist decrease amyloid pathology in the entorhinal cortex and learning and memory deficits in the 5xFAD mouse model of Alzheimer's disease. Neuropharmacology, 126, 128-141.

Baranger, K., Marchalant, Y., Bonnet, A. E., Crouzin, N., Carrete, A., Paumier, J. M., Py, N. A., Bernard, A., Bauer, C., Charrat, E., Moschke, K., Seiki, M., Vignes, M., Lichtenthaler, S. F., Checler, F., Khrestchatisky, M. \& Rivera, S. 2016. MT5-MMP is a new proamyloidogenic proteinase that promotes amyloid pathology and cognitive decline in a transgenic mouse model of Alzheimer's disease. Cell Mol Life Sci, 73, 217-36.

Bhattacharya, S., Haertel, C., Maelicke, A. \& Montag, D. 2014. Galantamine slows down plaque formation and behavioral decline in the 5XFAD mouse model of Alzheimer's disease. PLoS One, 9, e89454.

Boza-Serrano, A., Yang, Y., Paulus, A. \& Deierborg, T. 2018. Innate immune alterations are elicited in microglial cells before plaque deposition in the Alzheimer's disease mouse model 5xFAD. Sci Rep, 8, 1550.

Brandscheid, C., Schuck, F., Reinhardt, S., Schafer, K. H., Pietrzik, C. U., Grimm, M., Hartmann, T., Schwiertz, A. \& Endres, K. 2017. Altered Gut Microbiome Composition and Tryptic Activity of the 5xFAD Alzheimer's Mouse Model. J Alzheimers Dis, 56, 775788.

Buskila, Y., Crowe, S. E. \& Ellis-Davies, G. C. 2013. Synaptic deficits in layer 5 neurons precede overt structural decay in 5xFAD mice. Neuroscience, 254, 152-9. 
Callahan, M. J., Lipinski, W. J., Bian, F., Durham, R. A., Pack, A. \& Walker, L. C. 2001. Augmented senile plaque load in aged female beta-amyloid precursor protein-transgenic mice. Am J Pathol, 158, 1173-7.

Cechetto, D. F., Hachinski, V. \& Whitehead, S. N. 2008. Vascular risk factors and Alzheimer's disease. Expert Rev Neurother, 8, 743-50.

Citron, M., Westaway, D., Xia, W., Carlson, G., Diehl, T., Levesque, G., Johnson-Wood, K., Lee, M., Seubert, P., Davis, A., Kholodenko, D., Motter, R., Sherrington, R., Perry, B., Yao, H., Strome, R., Lieberburg, I., Rommens, J., Kim, S., Schenk, D., Fraser, P., St George Hyslop, P. \& Selkoe, D. J. 1997. Mutant presenilins of Alzheimer's disease increase production of 42-residue amyloid beta-protein in both transfected cells and transgenic mice. Nat Med, 3, 67-72.

Crouzin, N., Baranger, K., Cavalier, M., Marchalant, Y., Cohen-Solal, C., Roman, F. S., Khrestchatisky, M., Rivera, S., Feron, F. \& Vignes, M. 2013. Area-specific alterations of synaptic plasticity in the 5XFAD mouse model of Alzheimer's disease: dissociation between somatosensory cortex and hippocampus. PLoS One, 8, e74667.

De Jonghe, C., Esselens, C., Kumar-Singh, S., Craessaerts, K., Serneels, S., Checler, F., Annaert, W., Van Broeckhoven, C. \& De Strooper, B. 2001. Pathogenic APP mutations near the gamma-secretase cleavage site differentially affect Abeta secretion and APP C-terminal fragment stability. Hum Mol Genet, 10, 1665-71.

Devi, L., Alldred, M. J., Ginsberg, S. D. \& Ohno, M. 2010. Sex- and brain region-specific acceleration of beta-amyloidogenesis following behavioral stress in a mouse model of Alzheimer's disease. Mol Brain, 3, 34.

Devi, L. \& Ohno, M. 2010. Genetic reductions of beta-site amyloid precursor protein-cleaving enzyme 1 and amyloid-beta ameliorate impairment of conditioned taste aversion memory in 5XFAD Alzheimer's disease model mice. Eur J Neurosci, 31, 110-8.

Eimer, W. A. \& Vassar, R. 2013. Neuron loss in the 5XFAD mouse model of Alzheimer's disease correlates with intraneuronal Abeta42 accumulation and Caspase-3 activation. Mol Neurodegener, 8, 2.

Ferretti, M. T., Iulita, M. F., Cavedo, E., Chiesa, P. A., Schumacher Dimech, A., Santuccione Chadha, A., Baracchi, F., Girouard, H., Misoch, S., Giacobini, E., Depypere, H., Hampel, H., Women's Brain, P. \& the Alzheimer Precision Medicine, I. 2018. Sex differences in Alzheimer disease - the gateway to precision medicine. Nat Rev Neurol.

Fonseca, M. I., Zhou, J., Botto, M. \& Tenner, A. J. 2004. Absence of C1q leads to less neuropathology in transgenic mouse models of Alzheimer's disease. $J$ Neurosci, 24, 6457-65.

Giannoni, P., Arango-Lievano, M., Neves, I. D., Rousset, M. C., Baranger, K., Rivera, S., Jeanneteau, F., Claeysen, S. \& Marchi, N. 2016. Cerebrovascular pathology during the progression of experimental Alzheimer's disease. Neurobiol Dis, 88, 107-17.

Giannoni, P., Gaven, F., de Bundel, D., Baranger, K., Marchetti-Gauthier, E., Roman, F. S., Valjent, E., Marin, P., Bockaert, J., Rivera, S. \& Claeysen, S. 2013. Early administration of RS 67333, a specific 5-HT4 receptor agonist, prevents amyloidogenesis and behavioral deficits in the 5XFAD mouse model of Alzheimer's disease. Front Aging Neurosci, 5, 96.

Girard, S. D., Baranger, K., Gauthier, C., Jacquet, M., Bernard, A., Escoffier, G., Marchetti, E., Khrestchatisky, M., Rivera, S. \& Roman, F. S. 2013. Evidence for early cognitive impairment related to frontal cortex in the 5XFAD mouse model of Alzheimer's disease. J Alzheimers Dis, 33, 781-96.

Grinan-Ferre, C., Sarroca, S., Ivanova, A., Puigoriol-Illamola, D., Aguado, F., Camins, A., Sanfeliu, C. \& Pallas, M. 2016. Epigenetic mechanisms underlying cognitive impairment and Alzheimer disease hallmarks in 5XFAD mice. Aging (Albany NY), 8, 664-84. 
Gurel, B., Cansev, M., Sevinc, C., Kelestemur, S., Ocalan, B., Cakir, A., Aydin, S., Kahveci, N., Ozansoy, M., Taskapilioglu, O., Ulus, I. H., Basar, M. K., Sahin, B., Tuzuner, M. B. \& Baykal, A. T. 2018. Early Stage Alterations in CA1 Extracellular Region Proteins Indicate Dysregulation of IL6 and Iron Homeostasis in the 5XFAD Alzheimer's Disease Mouse Model. J Alzheimers Dis, 61, 1399-1410.

Harach, T., Marungruang, N., Duthilleul, N., Cheatham, V., Mc Coy, K. D., Frisoni, G., Neher, J. J., Fak, F., Jucker, M., Lasser, T. \& Bolmont, T. 2017. Reduction of Abeta amyloid pathology in APPPS1 transgenic mice in the absence of gut microbiota. Sci Rep, 7, 41802.

Herl, L., Thomas, A. V., Lill, C. M., Banks, M., Deng, A., Jones, P. B., Spoelgen, R., Hyman, B. T. \& Berezovska, O. 2009. Mutations in amyloid precursor protein affect its interactions with presenilin/gamma-secretase. Mol Cell Neurosci, 41, 166-74.

Hongpaisan, J., Sun, M. K. \& Alkon, D. L. 2011. PKC epsilon activation prevents synaptic loss, Abeta elevation, and cognitive deficits in Alzheimer's disease transgenic mice. $J$ Neurosci, 31, 630-43.

Hsiao, K., Chapman, P., Nilsen, S., Eckman, C., Harigaya, Y., Younkin, S., Yang, F. \& Cole, G. 1996. Correlative memory deficits, Abeta elevation, and amyloid plaques in transgenic mice. Science, 274, 99-102.

Iram, T., Trudler, D., Kain, D., Kanner, S., Galron, R., Vassar, R., Barzilai, A., Blinder, P., Fishelson, Z. \& Frenkel, D. 2016. Astrocytes from old Alzheimer's disease mice are impaired in Abeta uptake and in neuroprotection. Neurobiol Dis, 96, 84-94.

Jawhar, S., Trawicka, A., Jenneckens, C., Bayer, T. A. \& Wirths, O. 2012. Motor deficits, neuron loss, and reduced anxiety coinciding with axonal degeneration and intraneuronal Abeta aggregation in the 5XFAD mouse model of Alzheimer's disease. Neurobiol Aging, 33, 196 e29-40.

Jawhar, S., Wirths, O., Schilling, S., Graubner, S., Demuth, H. U. \& Bayer, T. A. 2011. Overexpression of glutaminyl cyclase, the enzyme responsible for pyroglutamate A \{beta\} formation, induces behavioral deficits, and glutaminyl cyclase knock-out rescues the behavioral phenotype in 5XFAD mice. J Biol Chem, 286, 4454-60.

Johnstone, M., Gearing, A. J. \& Miller, K. M. 1999. A central role for astrocytes in the inflammatory response to beta-amyloid; chemokines, cytokines and reactive oxygen species are produced. J Neuroimmunol, 93, 182-93.

Kimura, N., Takahashi, M., Tashiro, T. \& Terao, K. 2006. Amyloid beta up-regulates brainderived neurotrophic factor production from astrocytes: rescue from amyloid beta-related neuritic degeneration. J Neurosci Res, 84, 782-9.

Kook, S. Y., Hong, H. S., Moon, M., Ha, C. M., Chang, S. \& Mook-Jung, I. 2012. Abeta(1)()(4)(2)-RAGE interaction disrupts tight junctions of the blood-brain barrier via $\mathrm{Ca}(2)(+)-$ calcineurin signaling. J Neurosci, 32, 8845-54.

Landel, V., Millet, P., Baranger, K., Loriod, B. \& Feron, F. 2016. Vitamin D interacts with Esr1 and Igf1 to regulate molecular pathways relevant to Alzheimer's disease. Mol Neurodegener, 11, 22.

Liu, S., Liu, Y., Hao, W., Wolf, L., Kiliaan, A. J., Penke, B., Rube, C. E., Walter, J., Heneka, M. T., Hartmann, T., Menger, M. D. \& Fassbender, K. 2012. TLR2 is a primary receptor for Alzheimer's amyloid beta peptide to trigger neuroinflammatory activation. J Immunol, $188,1098-107$.

Maarouf, C. L., Kokjohn, T. A., Whiteside, C. M., Macias, M. P., Kalback, W. M., Sabbagh, M. N., Beach, T. G., Vassar, R. \& Roher, A. E. 2013. Molecular Differences and Similarities Between Alzheimer's Disease and the 5XFAD Transgenic Mouse Model of Amyloidosis. Biochem Insights, 6, 1-10.

MacPherson, K. P., Sompol, P., Kannarkat, G. T., Chang, J., Sniffen, L., Wildner, M. E., Norris, C. M. \& Tansey, M. G. 2017. Peripheral administration of the soluble TNF inhibitor 
XPro1595 modifies brain immune cell profiles, decreases beta-amyloid plaque load, and rescues impaired long-term potentiation in 5xFAD mice. Neurobiol Dis, 102, 81-95.

Marchetti, C. \& Marie, H. 2011. Hippocampal synaptic plasticity in Alzheimer's disease: what have we learned so far from transgenic models? Rev Neurosci, 22, 373-402.

Morello, M., Landel, V., Lacassagne, E., Baranger, K., Annweiler, C., Feron, F. \& Millet, P. 2018. Vitamin D Improves Neurogenesis and Cognition in a Mouse Model of Alzheimer's Disease. Mol Neurobiol.

Oakley, H., Cole, S. L., Logan, S., Maus, E., Shao, P., Craft, J., Guillozet-Bongaarts, A., Ohno, M., Disterhoft, J., Van Eldik, L., Berry, R. \& Vassar, R. 2006. Intraneuronal beta-amyloid aggregates, neurodegeneration, and neuron loss in transgenic mice with five familial Alzheimer's disease mutations: potential factors in amyloid plaque formation. JNeurosci, 26, 10129-40.

Orihuela, R., McPherson, C. A. \& Harry, G. J. 2016. Microglial M1/M2 polarization and metabolic states. Br J Pharmacol, 173, 649-65.

Perlmutter, L. S., Barron, E. \& Chui, H. C. 1990. Morphologic association between microglia and senile plaque amyloid in Alzheimer's disease. Neurosci Lett, 119, 32-6.

Rae, E. A. \& Brown, R. E. 2015. The problem of genotype and sex differences in life expectancy in transgenic AD mice. Neurosci Biobehav Rev, 57, 238-51.

Roddick, K. M., Roberts, A. D., Schellinck, H. M. \& Brown, R. E. 2016. Sex and Genotype Differences in Odor Detection in the 3xTg-AD and 5XFAD Mouse Models of Alzheimer's Disease at 6 Months of Age. Chem Senses, 41, 433-40.

Roddick, K. M., Schellinck, H. M. \& Brown, R. E. 2014. Olfactory delayed matching to sample performance in mice: sex differences in the 5XFAD mouse model of Alzheimer's disease. Behav Brain Res, 270, 165-70.

Schaeffer, E. L., Figueiro, M. \& Gattaz, W. F. 2011. Insights into Alzheimer disease pathogenesis from studies in transgenic animal models. Clinics (Sao Paulo), 66 Suppl 1, 45-54.

Scheuner, D., Eckman, C., Jensen, M., Song, X., Citron, M., Suzuki, N., Bird, T. D., Hardy, J., Hutton, M., Kukull, W., Larson, E., Levy-Lahad, E., Viitanen, M., Peskind, E., Poorkaj, P., Schellenberg, G., Tanzi, R., Wasco, W., Lannfelt, L., Selkoe, D. \& Younkin, S. 1996. Secreted amyloid beta-protein similar to that in the senile plaques of Alzheimer's disease is increased in vivo by the presenilin 1 and 2 and APP mutations linked to familial Alzheimer's disease. Nat Med, 2, 864-70.

Selkoe, D. J. \& Schenk, D. 2003. Alzheimer's disease: molecular understanding predicts amyloid-based therapeutics. Annu Rev Pharmacol Toxicol, 43, 545-84.

Spangenberg, E. E., Lee, R. J., Najafi, A. R., Rice, R. A., Elmore, M. R., Blurton-Jones, M., West, B. L. \& Green, K. N. 2016. Eliminating microglia in Alzheimer's mice prevents neuronal loss without modulating amyloid-beta pathology. Brain, 139, 1265-81.

Tohda, C., Urano, T., Umezaki, M., Nemere, I. \& Kuboyama, T. 2012. Diosgenin is an exogenous activator of 1,25D(3)-MARRS/Pdia3/ERp57 and improves Alzheimer's disease pathologies in 5XFAD mice. Sci Rep, 2, 535.

Wang, J., Tanila, H., Puolivali, J., Kadish, I. \& van Groen, T. 2003. Gender differences in the amount and deposition of amyloidbeta in APPswe and PS1 double transgenic mice. Neurobiol Dis, 14, 318-27.

Wu, Z., Guo, Z., Gearing, M. \& Chen, G. 2014. Tonic inhibition in dentate gyrus impairs longterm potentiation and memory in an Alzheimer's [corrected] disease model. Nat Commun, 5, 4159.

Wyss-Coray, T., Loike, J. D., Brionne, T. C., Lu, E., Anankov, R., Yan, F., Silverstein, S. C. \& Husemann, J. 2003. Adult mouse astrocytes degrade amyloid-beta in vitro and in situ. Nat Med, 9, 453-7. 


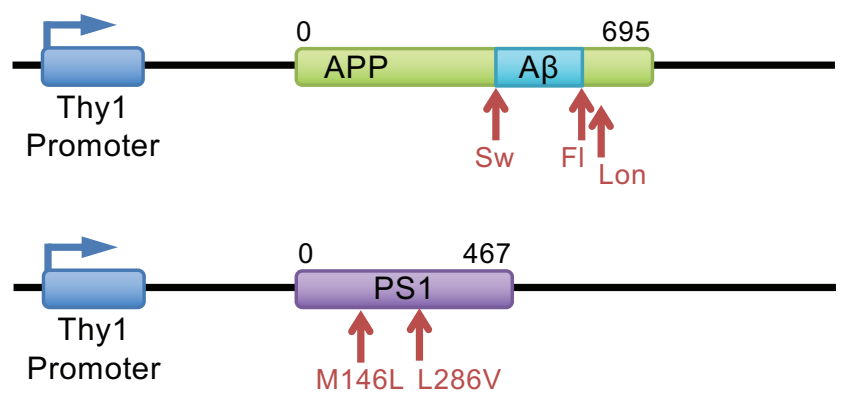

Figure 1 


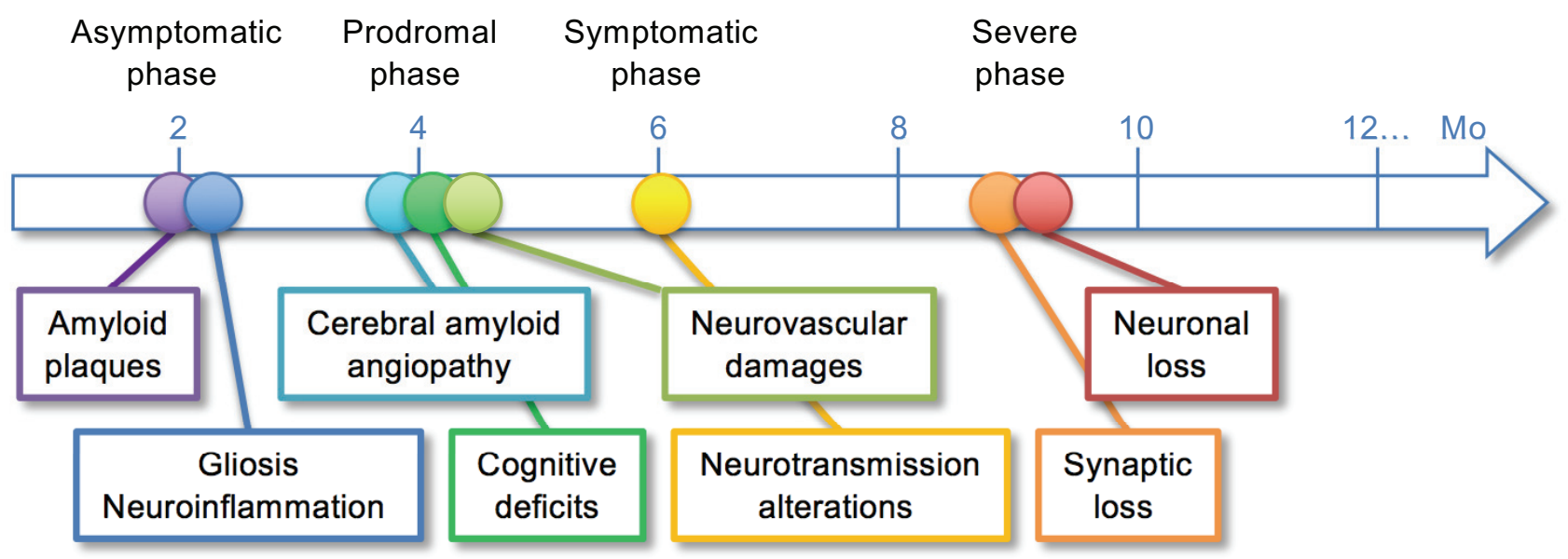

Figure 2 


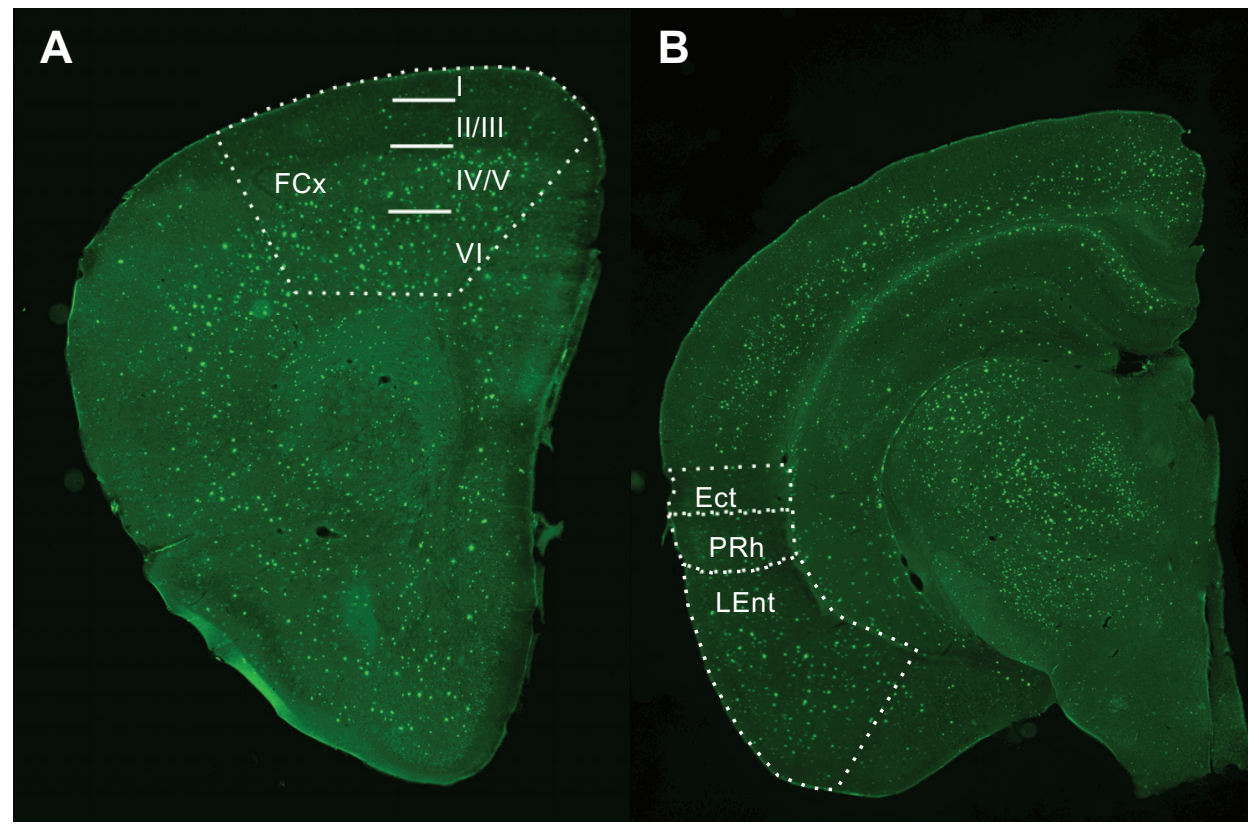

Figure 3 


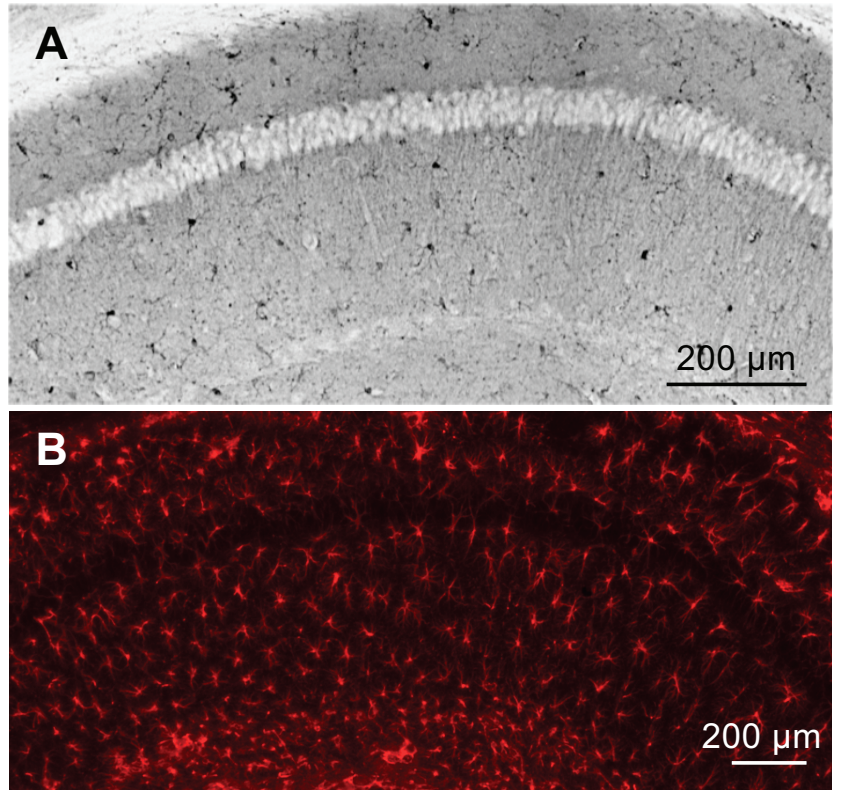

Figure 4 


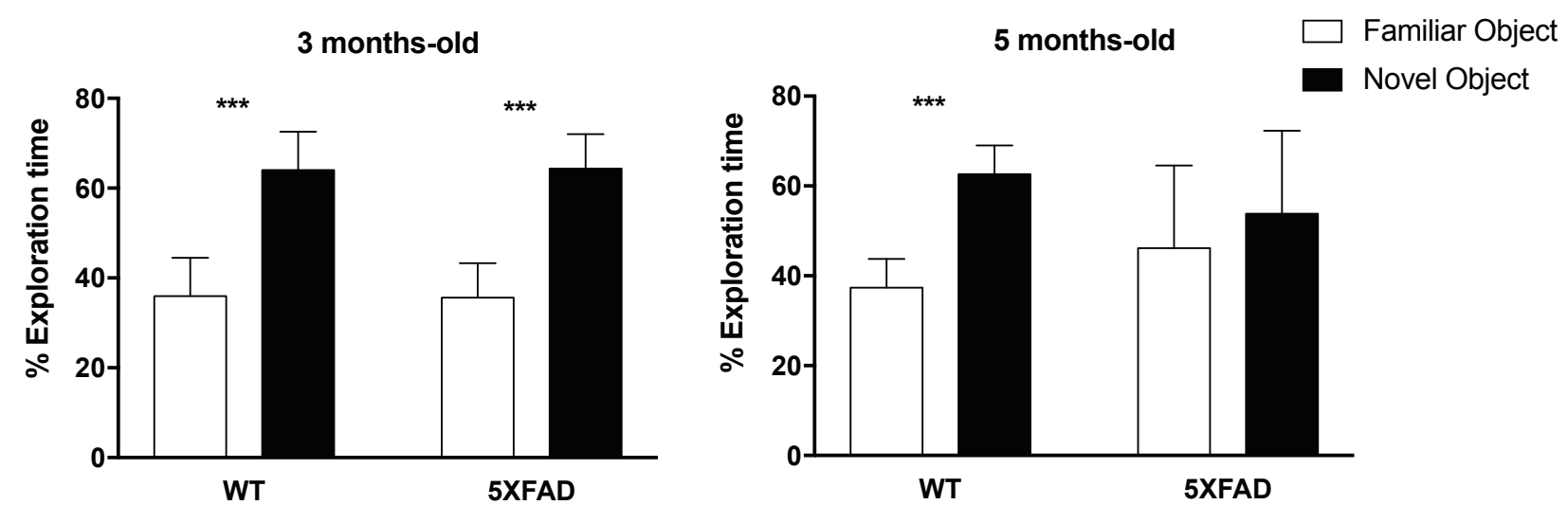

Figure 5 

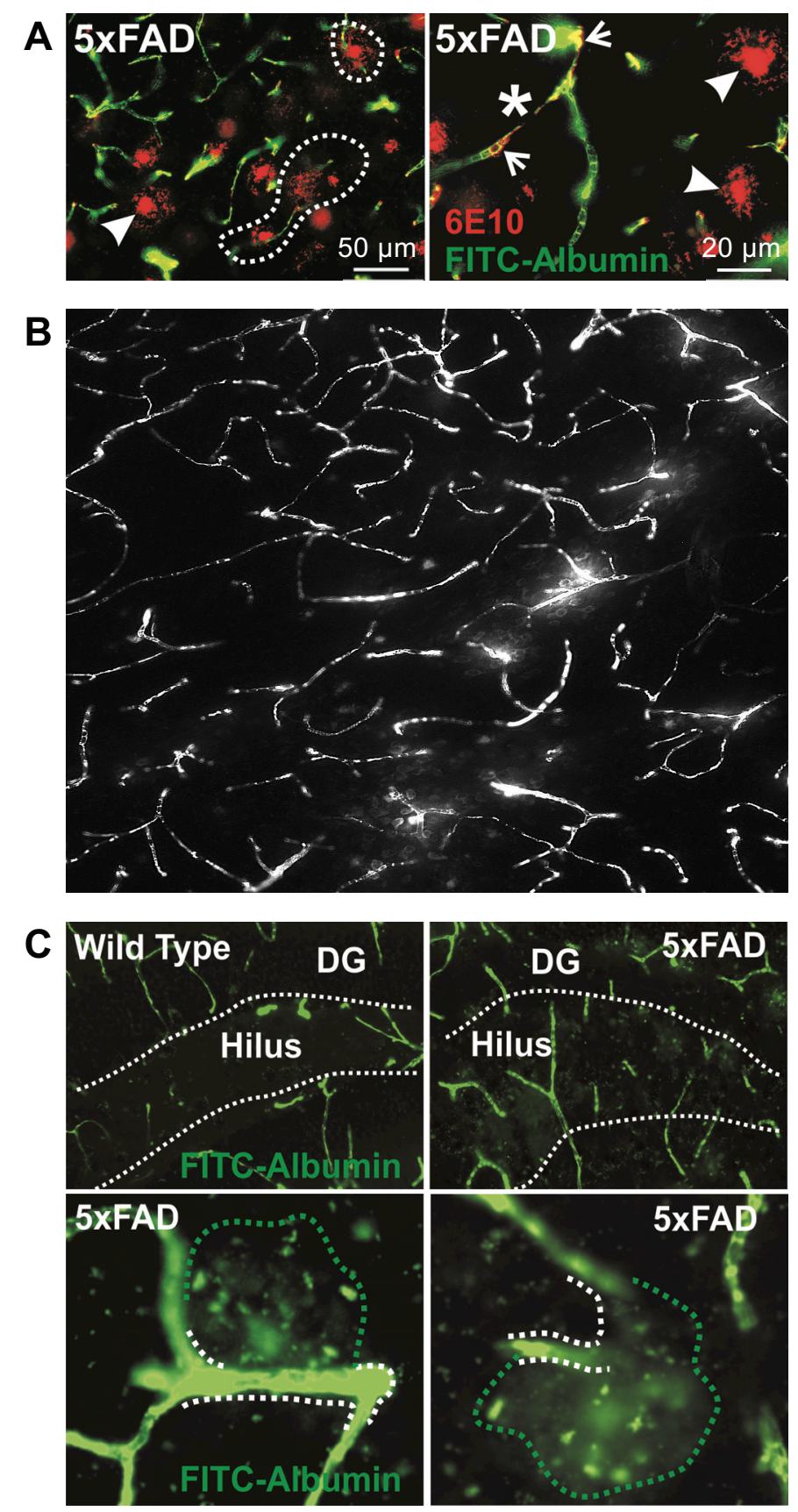

Figure 6 